\title{
Religious Group
}

National Cancer Institute

\section{Source}

National Cancer Institute. Religious Group. NCI Thesaurus. Code C103282.

A group of people characterized by the practice of a common religion. 